\title{
Cold Abscess of the Thigh in an Infant
}

\section{Mishra D, ${ }^{1}$ Mohta A, ${ }^{2}$ Arora $\mathrm{P}^{1}$}

\author{
${ }^{1}$ Department of Pediatrics \\ ${ }^{2}$ Department of Pediatric Surgery \\ Maulana Azad Medical College \\ Delhi, India
}

Corresponding Author

Devendra Mishra

Department of Pediatrics

Maulana Azad Medical College

Delhi, India

E-mail: drdmishra@gmail.com

\begin{abstract}
Local and minor adverse reactions to diphtheria-pertussis-tetanus (DPT) vaccination are usually mild and appear within 48 hours of vaccination. We herein report a rare association with intramuscular DPT injection and discuss pertinent issues. Primary tuberculous abscess was the final diagnosis.
\end{abstract}

\section{KEY WORDS}

Abscess, child, tuberculosis

Citation

Mishra D, Mohta A, Arora P. Cold Abscess of the Thigh in an Infant. Kathmandu Univ Med J 2013;41(1):86-87.

\section{INTRODUCTION}

Local adverse reactions following intramuscular injection of diphtheria-pertussis-tetanus (DPT) vaccine are usually mild, and appear within 48 hours of vaccination. Common side-effects seen are mild soreness, erythema, swelling or indurations at injection site. In some cases mild fever may follow. Rarely, one may see fatigue, malaise, headache, arthralgia, myalgia, urticaria, anaphylactoid reaction and hypotonic-hyporesponsive episodes and convulsions within two-three days of vaccination. We herein report a rare association with intramuscular DPT injection and discuss pertinent issues.

\section{CASE-REPORT}

In April 2007, an eight months-old female child presented to the outpatient department of Chacha Nehru Bal Chikitsalaya, with a swelling on anterolateral aspect of right thigh. The swelling had gradually developed at the site of intramuscular DPT injection within five days after its administration. It was $3.5 \times 1.5 \mathrm{~cm}$ in size, not warm, nontender, fluctuant, non-pulsatile and in the subcutaneous plane (Fig. 1). This child had no prior complaints and was thriving well, had no history of repeated infections, and had appropriate weight-for-age. Fine needle aspiration cytology (FNAC) of the swelling showed caseating granulomas suggestive of tuberculosis. Staining for acidfast bacilli was positive. This child had no history of contact with tuberculosis. X-ray of hip joints (Fig. 2), thigh and spine showed no bony abnormality or evidence of chronic osteomyelitis. On further detailed investigations (Chest $\mathrm{X}$-ray, gastric aspirate for AFB, erythrocyte sedimentation rate, and ultrasound abdomen), no evidence of tuberculosis at any other site was found and, screening of caregivers was also non-contributory. Child had received Bacillus-Calmette-Guérin (BCG) at birth (on left shoulder) and Mantoux test (with 1 TU Purified protein derivative) was positive. Antitubercular therapy was started with four drugs but the swelling did not subside over the next 


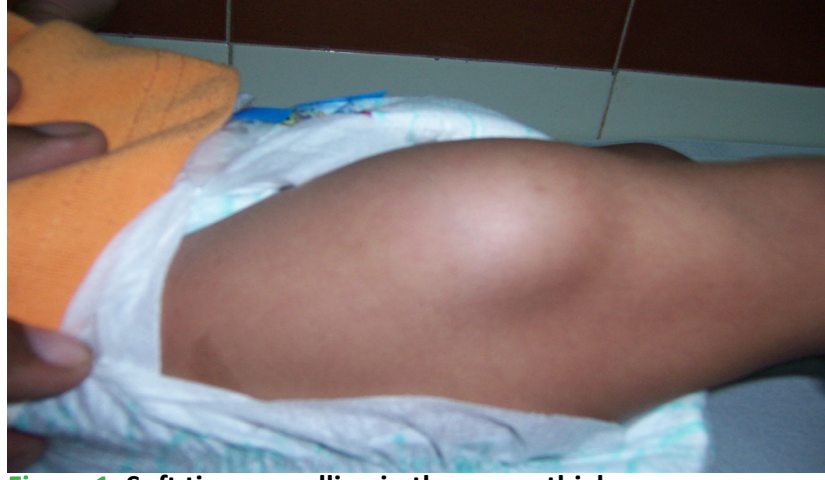

Figure 1. Soft tissue swelling in the upper thigh.

six weeks. Antigravity drainage of the swelling was done. Antitubercular therapy was continued for six months and follow up examination showed no swelling and a wellthriving child with adequate weight gain.

\section{DISCUSSION}

Cold abscesses, abscesses without systemic toxic signs and with few focal inflammatory signs, may be present in immunodeficiency disorders, deep mycoses, malnutrition and other infectious diseases, in addition to tuberculosis. ${ }^{1}$ Tuberculous infection is the most common cause of such abscesses. $^{2-4}$ Tuberculosis involving the soft tissue from adjacent bone or joint is well recognized and cold abscess of the psoas muscle is generally one of the characteristic manifestations of spinal tuberculosis. ${ }^{5}$ Tuberculosis of the pubis presenting as a cold abscess of thigh has also been reported. ${ }^{4}$ As this child had no evidence of tuberculosis of spine, pubis or any other parts of body, we could not explain the origin of infection and also its occurrence at the site of DPT injection.

Literature search revealed quite a few reports from the 1950s, indicating that primary tuberculous gluteal abscess can be syringe-transmitted. ${ }^{5-7}$ Four cases occurred in a single hospital where a nurse had developed pulmonary tuberculosis and had given multiple muscular injections to the affected children. 5,6 Debré et al reported an epidemic in a nursery in 1951. A needle and a syringe were contaminated with Mycobacterium tuberculosis from a child with a tuberculous gluteal abscess who had been given an injection of penicillin, and the same syringe was used on other infants. ${ }^{5,7}$ However, during that era, reuse after sterilization of needles and syringes was the norm, and deficiency in sterilization might explain the cause of these infections. No such cause could be identified in our patient. Non-tubercular cold abscess of the thigh following intramuscular injection and non-tubercular cold abscess of psoas muscle have previously been reported. ${ }^{8,9}$ Another differential diagnosis can be tuberculous myositis, but the lesion was in the subcutaneous tissue, superficial to the muscle plane. ${ }^{5}$ Primary tuberculous gluteal abscess, without bone involvement, has recently been reported in infancy and is a distinct possibility in this child.

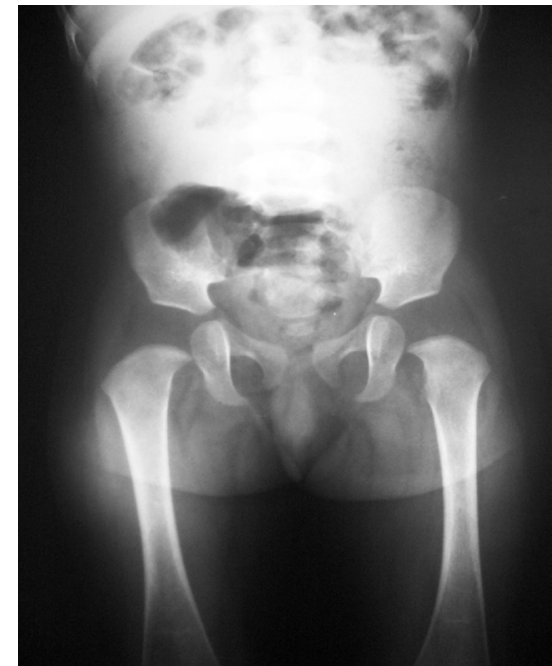

Figure 2. AP X-ray of both hips shows no abnormalities. The cartilage space and articular margins are intact.

We wish to sensitize both the pediatricians and the pediatric surgeons to the possibility of a primary tuberculous abscess, even when the joints and bone adjacent to a superficial abscess are intact.

\section{REFERENCES}

1. Jackson R, Stephens L, Kelly P. Cold subcutaneous abscesses. J Natl Med Assoc 1990; 82: 733-736.

2. Terada $\mathrm{Y}$, Matsunobe S, Nemoto T, Tsuda T, Tonomura S. Contents of chest wall cold abscess flowing into the epidural space in military tuberculosis. Chest 1992; 101: 590-591.

3. Yoshioka K. Multiple pleural tuberculomas associated with a cold abscess of the hip. Respiration 1995; 62: 46-48.

4. Gilroy BP. Tuberculosis of pubis presenting as a cold abscess of the thigh. Br Med J 1955; 2 (4943): 832-33.

5. Abdelwahab IF, Bianchi S, Martinoli C, Klein M, Hermann G. Atypical extraspinal musculoskeletal tuberculosis in immunocompetent patients: Part II, tuberculous myositis, tuberculous bursitis, and tuberculous tenosynovites. Can Assoc Radiol J 2006; 57(5): 278-286.

6. Heycock JB, Noble TC. Four cases of syringe-transmitted tuberculosis. Tubercle 1961; 8: 23-6.

7. Debré R Brussaud, Canlorbe P. Vaccine associated tuberculosis in children. Arch Fr Pediatr 1951;8:49-50.

8. Lambotte-Legrand J, Lambotte-Legrand C. Cold abscess of the thigh following an intramuscular injection. Arch Fr Pediatr 1951; 8(1): 6566.

9. Lee SS, Chan YS, Chao CY, Fu TS, Ueng SW. Non-tubercular cold abscess of the psoas muscle-an unusual manifestation of colocutaneous fistula. Arch Orthop Trauma Surg 2000; 120: 224-25. 